\title{
A Study On A Treatment System For Hyperhidrosis And A Solution That Combines Focused Ultrasound And Ultrasound Imaging Technology
}

\author{
Jong-Chul Park, Woo-Won Jeong, Min-Sung Kim* \\ Dept. of Information \& Communications Engineering, TongMyong University, Busan, 48520, Korea \\ *Corresponding author. Tel.: +82-10-8618-3879; Email address: minsung@ tu.ac.kr
}

Article History:Received:11 February 2021; Accepted: 27 March 2021; Published online: 5 April 2021

\begin{abstract}
In this paper, for the treatment of axillary hyperhidrosis, we propose a driving algorithm for diagnosing the location / distribution / degree of sweat glands using ultrasound images and implementing a treatment device using high intensity focused ultrasound. Currently, treatment for axillary hyperhidrosis uses surgery to cut the skin itself or remove the sweat glands using an incision, but this has the disadvantage of scarring and long recovery time. To solve this, we propose a treatment device using high intensity focused ultrasound and a driving algorithm and a method for implementing a user interface environment.
\end{abstract}

Keywords: Ultrasound, Hyperhidrosis, Handpiece, Sweat Gland, HIFU.

\section{Introduction}

Fluid and hyperhidrosis is a disease that offends people around and interferes with social life by emitting a peculiar smell due to the abnormalities of the sweat glands, and the main cause of it is related to the sweat glands hidden under the armpit. Treatment for fluid and hyperhidrosis involves surgery of cutting off the skin and using the incision to remove sweat glands, but this method has disadvantages of scarring and long recovery time, so it is not preferred recently. High-intensity focused ultrasound (HIFU) and radiofrequency (RF) are used for non-invasive skin tightening[1].

To solve this problem, this paper diagnoses the position/distribution/precision of sweat glands using ultrasonic images and presents a plan for realizing the treatment and driving algorithm and user interface environment using high-intensity concentrated ultrasound.

With increasing public concern about facial wrinkles and loss of skin elasticity due to aging, diverse devices have been proposed as treatment modalities for facial wrinkles and laxity[2]. Recently, ultrasonography was introduced as a new treatment modality for therapeutic and cosmetic purposes. High-intensity focused ultrasonography (HIFU) may be the best symbol of this technique. It is based on the principle of induction of tissue damage and regeneration of the target area selectively via coagulation by generating microthermal injury lesions through the accumulation of high-frequency ultrasonography beams at the specific tissue site without any damage to the epidermis and adjacent tissue[3][4]. High Intensity Ultrasound is a therapeutic ultrasound, many studies are under way and the positive treatment effect of high intensity concentration ultrasound is being demonstrated[5][6]. High-intensity focused ultrasound (HIFU) for non-invasive treatment with a range of internal pathologies including cancers of major organs and cerebral pathologies is in exponential growth[7].

Treatment for fluid and hyperhidrosis is doing the surgery of cutting off the skin and using the incision to remove sweat glands, but it is difficult to return to daily life after the surgery and has the disadvantage of scarring. Medical devices using laser or RF to make up for these surgical shortcomings have emerged, but the recurrence rate is high and there might be side effects during the procedure. Recently, a treatable technology has been developed using microwave technology, but it is difficult to apply it in practice due to the inherent risks of microwave technology and the disadvantages of destroying tissues other than sweat glands[8].

\section{Materials and methods}

2.1 Axillary Hyperhidrosis Diagnosis System

For the treatment of fluid and hyperhidrosis, a diagnosis is needed to accurately identify the current sweat glands in the skin. Therefore, an ultrasonic image probe that can check the position of the sweat glands during the procedure is added inside the handpiece so that the position of the sweat glands can be checked accurately.
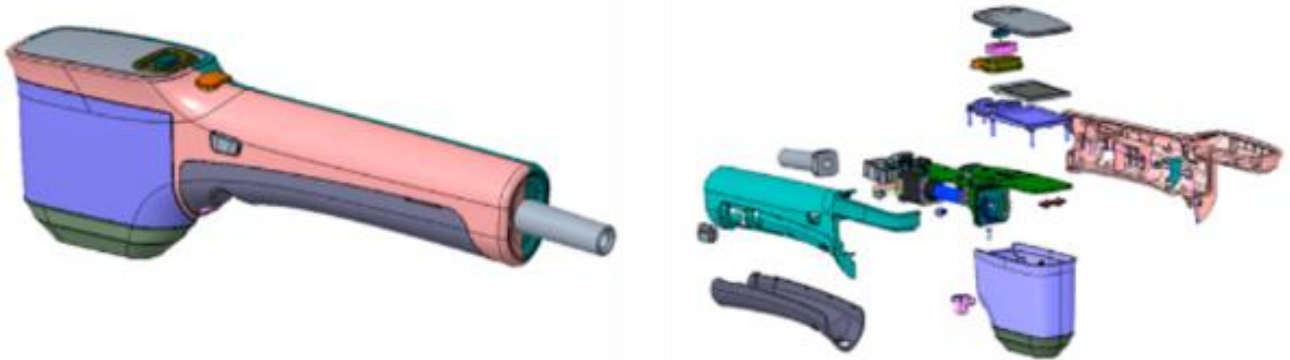

Figure 1. Handpiece design

*Corresponding author: Min-Sung Kim*

Dept. of Information \& Communications Engineering, TongMyong University, Busan, 48520, Korea

${ }^{*}$ Corresponding author. Tel.: +82-10-8618-3879; Email address: minsung@tu.ac.kr 


\subsection{HIFU ultrasonic focal point driving algorithm}

The location of the sweat glands is identified through ultrasonic images and other components are developed to accurately target only the sweat glands without damage. The location of the ultrasonic glands can be adjusted by taking into account the irregular sweat glands. The ultrasonic transducer is controlled so that it can move to the $\mathrm{x}$-axis at regular intervals and move to the y-axis to control the depth of treatment.

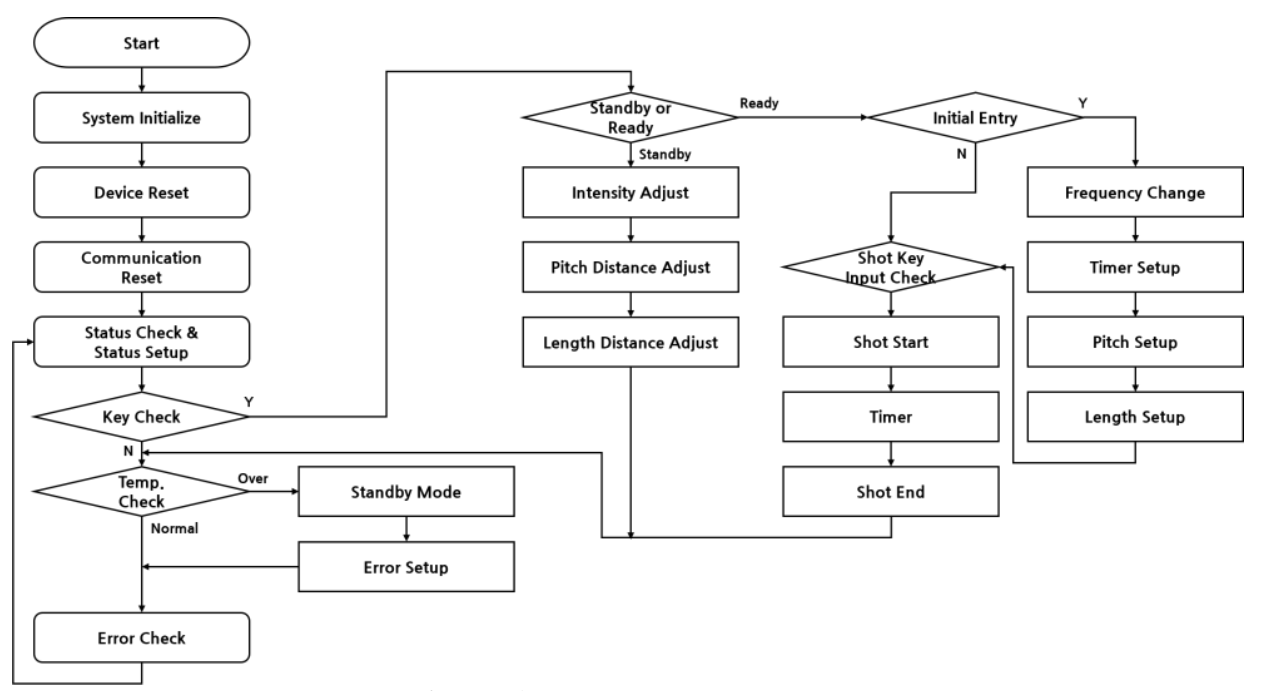

Figure 2. Flow Chart of Device
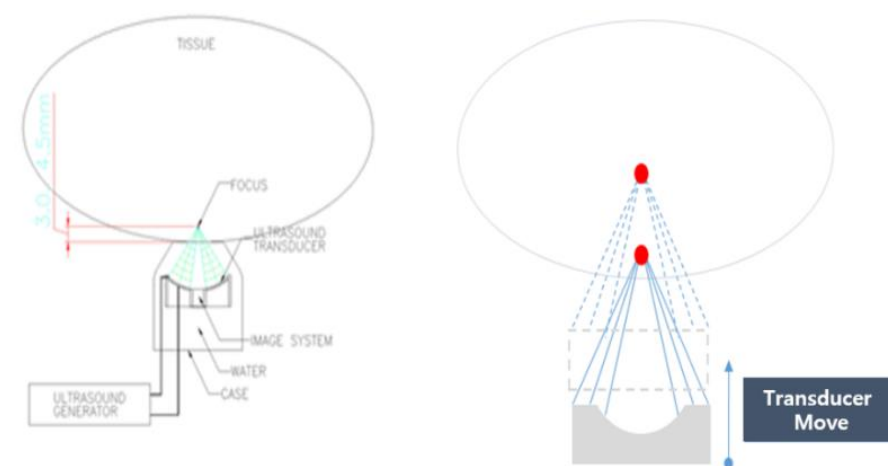

Figure 3. High intensity focused ultrasound device and position control technology schematic

\subsection{HIFU ultrasonic focal point driving algorithm}

The 10.1-inch touchscreen LCD is applied to provide a wide range of user interfaces, which are designed to prevent malfunctions and recognize the status of equipment at a glance. Unnecessary elements were excluded and composed of objects faithfully to function.

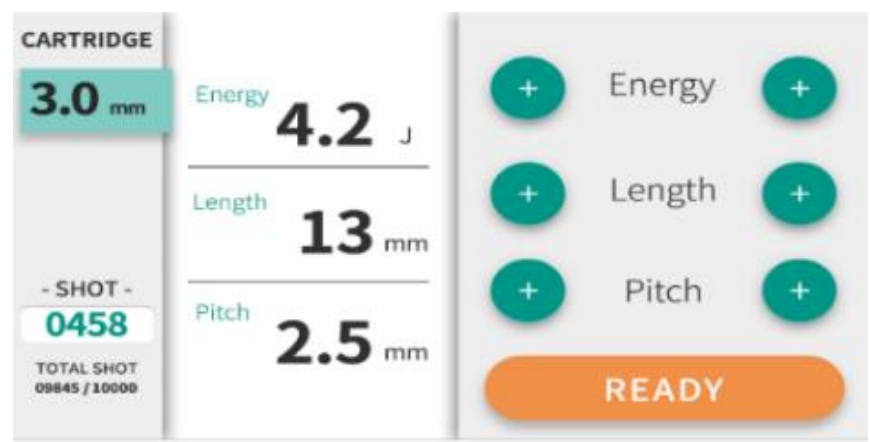

Figure 4. Expected user interface design

\section{Results}

The main performance of the treatment device using focused ultrasound can be expressed by the intensity of 
the therapeutic energy and the focal temperature. Accordingly, the intensity of ultrasonic waves was repeatedly measured 100 times using an ultrasonic radiation force meter. As a result of the measurement, the maximum intensity of the ultrasound was measured as 2.65 , the minimum intensity was 2.4 , and the average was 2.53 .

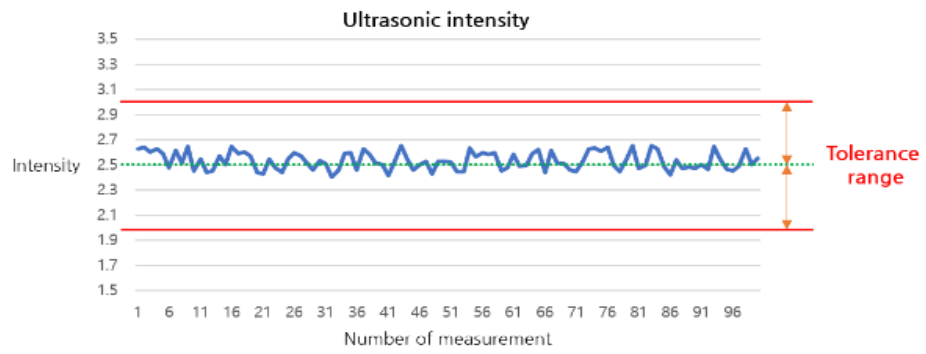

Figure 5. Ultrasonic intensity

Table 1. Ultrasonic intensity

\begin{tabular}{c|c|c}
\hline & Ultrasonic intensity $\left(\mathrm{J} / \mathrm{cm}^{2}\right)$ & $\operatorname{Error}(\%)$ \\
\hline Max. Value & 2.65 & 6.0 \\
\hline Min. Value & 2.40 & 3.9 \\
\hline Average & 2.53 & 1.3 \\
\hline
\end{tabular}

In addition, the focal temperature of the therapeutic ultrasound should be between $50{ }^{\circ} \mathrm{C}$ and $60{ }^{\circ} \mathrm{C}$, the temperature change in the area excluding the focal area should be $10{ }^{\circ} \mathrm{C}$ or less. Table 2 and Figure 2 show the results of 100 experiments of temperature measurement.

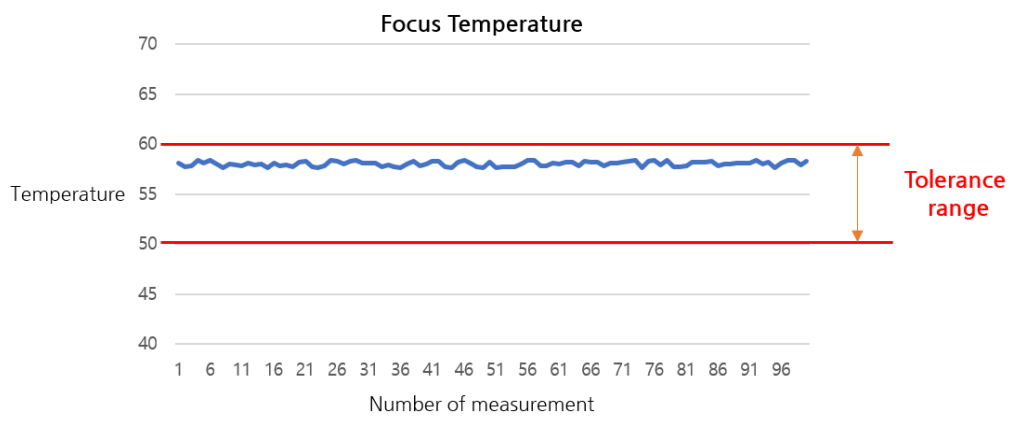

Figure 6. Focus Temperature

Table 2. Focus Temperature

\begin{tabular}{c|c|c}
\hline & Focus Temp. $\left({ }^{\circ} \mathrm{C}\right)$ & Ambient temp. change value $\left({ }^{\circ} \mathrm{C}\right)$ \\
\hline Max. Value & 58.4 & 9.5 \\
\hline Min. Value & 57.6 & 0.8 \\
\hline Average & 58.0 & 5.4 \\
\hline
\end{tabular}

An experiment was conducted on the degree of error in the focal distance, one of the main functions of the super-sonic band. The error shall be formed within $\pm 1 \mathrm{~mm}$ of the $3 \mathrm{~mm}$ reference, but the test results show that accurate control is possible by measuring between $2.47 \mathrm{~mm}$ and $3.39 \mathrm{~mm}$.

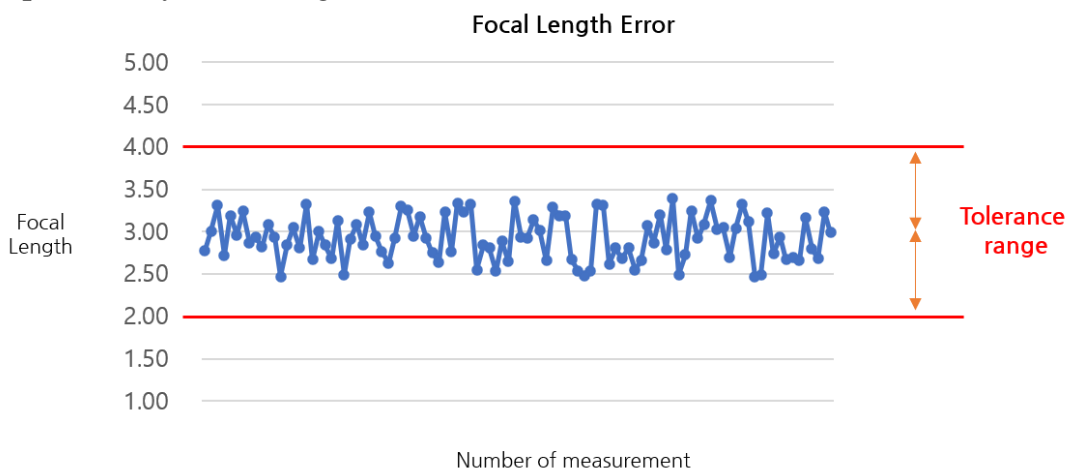


Figure 5. Focal Length Error

\section{Discussion}

For the treatment of fluid and hyperhidrosis, the position/distribution/precision of sweat glands were diagnosed using ultrasonic images and a driving algorithm was proposed to implement a treatment device using high-intensity concentrated ultrasound, at the same time the prototypes were produced and experimented. The results of the experiment were showed in Tables 1 and 2.

\section{Conclusions}

The algorithms and devices proposed in this paper are expected to be developed into non-invasive medical technology through the development of future applications for chemotherapy devices such as skin cancer, thyroid cancer and other medical fields by applying intensive care to specific areas of the human body.

\section{References}

1. Dong Hye Suh, JeongHwee Choi, Sang Jun Lee, Ki-HeonJeong, Kye Yong Song \& Min Kyung Shin, Comparative histometric analysis of the effects of high-intensity focused ultrasound and radiofrequency on skin : Journal of Cosmetic and Laser Therapy, Volume 17, 2015 - Issue 5, https://doi.org/10.3109/14764172.2015.1022189

2. Lee, Woo-Cheol, Ko, Yun-Seok, A Study on the Development of High-intensity focused Ultrasound Device for the Beauty Treatment Health Care : The Journal of the Korea institute of electronic communication sciences, Volume 11, Issue 12, Pages.1259-1264, https://doi.org/10.13067/JKIECS.2016.11.12.1259

3. SuJin Oh, Won Seon Koh, Jae Min Shin, JeongEun Kim, Joo Yeon Ko, Facial Rejuvenation with Highintensity Focused Ultrasonography : Korean Journal of Dermatology, Vol. 54, No. 4, pp.249-254

4. Hui Zhu, Kun Zhou, Lian Zhang, ChengbinJin, Song Peng, Wei Yang, Kequan Li, Haibing Su, WenzhiChen, Jin Bai, Feng Wu, Zhibiao Wang, High intensity focused ultrasound (HIFU) therapy for local treatment of hepatocellular carcinoma: Role of partial rib resection : European Journal of Radiology, Volume 72, Issue 1, October 2009, Pages 160-166, https://doi.org/10.1016/j.ejrad.2008.07.003

5. Su Mi Noh, Tae WoongJeong, SeongChul Kim, Kyung Won Shin, Joo Young Kim, Hong Ho Choi, Fundamental Study on the Change of RBC Count by Focused Ultrasound Sonication Condition : Journal of the Korean Society of Radiology, 2018, page 49

6. S.Y. Choi, Y.A. No, S.Y. Kim, B.J. Kim, M.N. Kim, Tightening effects of high-intensity focused ultrasound on body skin and subdermal tissue: a pilot study : Journal of The European Academy of Dermatology and Venereology, Volume30, Issue9 , September 2016, Pages 1599-1602, https://doi.org/10.1111/jdv.13713

7. Torsten Bove, Tomasz Zawada, JørgenSerup, Alexander Jessen, Mattia Poli, High-frequency (20$\mathrm{MHz}$ ) high-intensity focused ultrasound (HIFU) system for dermal intervention: Preclinical evaluation in skin equivalents : Skin Research \& Technology Volume25, Issue2 March 2019 Pages 217-228, https://doi.org/10.1111/srt.12661

8. NaohikoSenoo, Hiroyuki Ushijima, Jun Suzuki, Kiyoshi Yoshinaka, Juno Deguchi, Shu Takagi, Tetsuro Miyata, and Yoichiro Matsumoto, Development of HIFU Treatment for Lower Extremity Varicose Veins : AIP Conference Proceedings 1359, 30 (2011), https://doi.org/10.1063/1.3607878 